\title{
Diffusion on a heptagonal lattice
}

\author{
Seung Ki Baek, Su Do Yi, and Beom Jun Kim* \\ Department of Physics, BK21 Physics Research Division, and Institute of Basic Science, \\ Sungkyunkwan University, Suwon 440-746, Republic of Korea
}

\begin{abstract}
We study the diffusion phenomena on the negatively curved surface made up of congruent heptagons. Unlike the usual two-dimensional plane, this structure makes the boundary increase exponentially with the distance from the center, and hence the displacement of a classical random walker increases linearly in time. The diffusion of a quantum particle put on the heptagonal lattice is also studied in the framework of the tight-binding model Hamiltonian, and we again find the linear diffusion like the classical random walk. A comparison with diffusion on complex networks is also made.
\end{abstract}

PACS numbers: 05.40.Fb, 89.75.Hc, 66.30.-h, 05.60.Gg

The transport phenomena have been extensively studied on various spatial structures to reveal the characteristic thermal and electrical properties of materials. The random walk is one of the classical topics for studying those phenomena in the nonequilibrium statistical physics [1], and the transport on highly disordered systems such as complex networks is also of recent research interest [2, 3, 4]. Since the average path length is often greatly reduced in complex networks, one can easily expect that a particle diffuses there faster than on the ordinary regular lattice structures.

Recently, the surface with a constant negative Gaussian curvature is under active investigation to study geometrical effects on the critical phenomena [5, [6]. A negative Gaussian curvature means that the surface locally looks like a uniform saddle everywhere, with the resulting surface getting highly curled as one moves outward [7]. Consequently, the boundary length of a circle with radius $r$ is given by $2 \pi \sinh r$, rather than by $2 \pi r[8]$, which means in turn that the characteristic path length increases only logarithmically with the system size $N[6]$. For a positively curved surface, the system becomes eventually closed, like a sphere, where the magnitude of curvature has to approach zero in the thermodynamic limit. Accordingly, there is little reason to study this case separately. In contrast, surfaces of a constant negative Gaussian curvature can be extended indefinitely, which makes such a geometry apt for studying novel physical properties. Furthermore, the development of nanotechnology can make it possible to construct such a structure in reality, and the physical properties discovered here are also of practical importance. There already exist theoretical interests in negatively curved nanostructures with a very large surface area embedded in a limited volume [9].

It has been reported that the hyperbolic Brownian motion has a limited direction [10] with a constant outward drift [1]. Such nontrivial behaviors are nothing mysterious and directly related to the exponentially increasing length of a boundary: Since there is always a larger num-

${ }^{*}$ Corresponding author, E-mail: beomjun@skku.edu
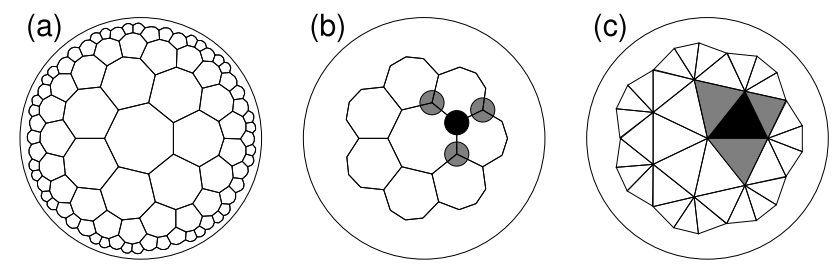

FIG. 1: (a) Heptagonal lattice represented on the Poincaré disk with the total number of levels $L=4$. The metric is given in such a way that all heptagons are congruent and the circle indicates the points at infinity. (b) Random walk on the heptagonal lattice where a walker at the black dot has three choices (colored in gray) at each time. (c) In the dual lattice, walking around a heptagon is equivalent to the reflection of a triangle.

ber of points outside than inside by some constant proportion, a random walker tends to move outward, which allows the random walker only a limited direction [12]. However, the validity of such a simple geometric understanding of the fast classical diffusion needs to be taken carefully if one compares the classical diffusion with the quantum one, which composes the main motivation of this Brief Report.

In this work, we present how to simulate the random walk on such a geometry and reconfirm the linear classical diffusion for a heptagonal lattice structure. The quantum diffusion of a particle is then investigated in comparison both with the classical diffusion behavior and with Ref. 2] in which the quantum diffusion time has been shown to scale as $\tau_{q} \sim N^{\alpha-1}$ while $\tau_{c} \sim N^{\alpha}$ for the classical diffusion. Also in Ref. [3], quantum mechanical transport on graphs has been shown to be faster than the classical one, except on some finite treelike graphs. In comparison, $\tau_{q} \sim \tau_{c}$ is revealed in the present work on the heptagonal lattice geometry. It is remarkable that one can drastically accelerate the transport by introducing heptagonal plaquettes to the lattice [9].

Let us describe the structure of the heptagonal lattice. Similarly to the two-dimensional (2D) plane which can be covered by congruent triangles, squares, and hexagons, a negatively curved surface can be tiled by polygons 
through the hyperbolic tessellation [13]: Suppose a lattice where $q$ regular $p$-gons meet at each vertex. For example, the triangular lattice is denoted as $p=3$ and $q=6$. It is known that the tiling with given $p$ and $q$ covers the negatively curved surface if $(p-2)(q-2)>4$. If we choose its basic element as the simplest polygon, the regular triangle, at least seven triangles should meet at each vertex leading to $\{p, q\}=\{3,7\}$. Taking its dual lattice, we obtain the heptagonal lattice structure with $\{p, q\}=\{7,3\}$. The resulting structure can be most suitably represented in the Poincaré disk [14] as in Fig. 1(a). Since the heptagons are located in a concentric fashion, we denote the central heptagon as the first level. Accordingly, its seven nearest-neighbor heptagons constitute the second level, and the next nearest ones the third level, and so on. Figure 1(a) thus describes the heptagonal lattice up to the level $l=4$. Let $H(l)$ be the set of heptagons in the $l$ th level. The set of vertices on the outward boundary of $H(l)$ is called the $l$ th layer. As one can see, this structure uniformly and completely fills the surface and provides an adequate tool for studying physics of the negative Gaussian curvature. For numerical calculations, we may use the heptagonal lattice constructed up to some finite level, $L$, and capture the finite size effects by varying $L$.

We first study numerically the random walk problem in our heptagonal lattice structure, and the results are compared with numerical solutions of the diffusion equation for the hyperbolic geometry. A possible way of simulating a random walker on the heptagonal lattice is to identify the connection structure first and then let a particle move along the edges. In Fig. 2(a), the position of the random walker measured by the level $l$ is shown as a function of the time step $n$ for the heptagonal lattices of sizes $L=7,10$, and 14. It is clearly shown that the random walker drifts away from the starting position $l=1$ linearly in time. As the lattice becomes larger (as $L$ is increased), the linear diffusion regime becomes extended, indicating $\langle l\rangle \propto n$ in the thermodynamic limit. One drawback of this approach is that the number of points increases exponentially as we add concentric layers. The memory constraint restricts the distance from the origin, and therefore the results severely suffer from finiteness of the model system under consideration.

A better alternative is obtained from the hyperbolic tessellation. Since the heptagonal lattice is dual to $\{3,7\}$, the transition between the neighboring points coincides with reflecting a hyperbolic triangle [see Fig. 1(c)]. Since the Poincaré disk can be identified with a unit disk on the complex plane, we start from a regular triangle $\left(z_{1}, z_{2}, z_{3}\right)$ where $z_{i}$ 's are complex numbers. Let one of the vertices, say $z_{1}$, be located at the origin. Setting the interior angle at that point to be $2 \pi / 7$, the triangle is regular only when $\left|z_{1}-z_{2}\right|=\left|z_{1}-z_{3}\right| \approx 0.49697$, as this surface has its own intrinsic length scale, i.e., the curvature [14]. If a triangle $\left(z_{i}, z_{j}, z_{k}\right)$ is reflected around the edge along $z_{i}$ and $z_{j}$, we get a new triangle $\left(z_{i}, z_{j}, z_{k}^{\prime}\right)$ with $z_{k}^{\prime}=w+\xi^{2} /\left(\overline{z_{k}}-\bar{w}\right)$, where $w=\left(\left|z_{i}\right|^{2} z_{j}-z_{i}\left|z_{j}\right|^{2}-z_{i}+z_{j}\right) /\left(\overline{z_{i}} z_{j}-z_{i} \overline{z_{j}}\right)$,
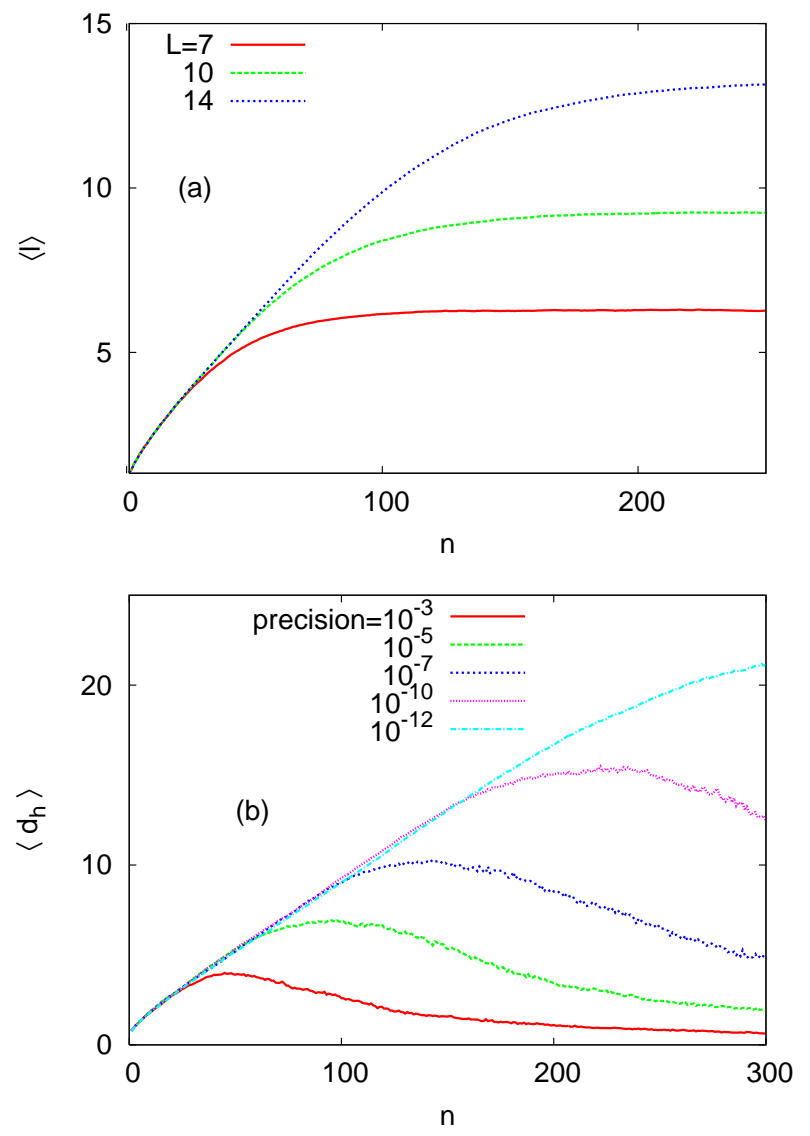

FIG. 2: (Color online) (a) Average level $\langle l\rangle$ of the random walker versus the time $n$. The heptagonal lattice of the size $L$ is first built, and we perform random walks as in Fig. 1(b). We see $\langle l\rangle \propto n$ for small $n$, while $\langle l\rangle$ eventually saturates as the walker approaches the external boundary. (b) Average distance $\left\langle d_{h}\right\rangle$ from the origin versus the time $n$, obtained from the method in Fig. 1(c). The deviation from the linear behavior is not due to the finiteness of the lattice as in (a), but due to accumulated numerical errors. All the averages were taken from $10^{3}$ independent realizations.

$\xi=\left|w-z_{i}\right|=\left|w-z_{j}\right|$, and $\bar{z}$ is the complex conjugate of $z$. The center of a triangle, $z$, has the hyperbolic distance from the origin by $d_{h}(z)=\ln [(1+|z|) /(1-|z|)]$. Note that $d_{h}$ diverges to infinity as $|z| \rightarrow 1$, which is consistent with the definition of the Poincaré disk. The result is depicted in Fig. 2(b), which clearly shows that the distance is linearly proportional to time. The numerical inaccuracy becomes larger as we repeat the reflection of triangles. In order to confirm the origin of the deviation from the linear behavior $\left\langle d_{h}\right\rangle \sim n$ at large $n$, we intentionally assign numerical precision and observe how $\left\langle d_{h}\right\rangle$ depends on it. In Fig. 2(b), it is seen that as we use better numerical precision, the linear regime becomes more extended, indicating that the deviation from the linear behavior originates from the simple artifact of the numerical accuracy. Beyond the deviation point of each curve in Fig. 2, the numerical values are not trust- 


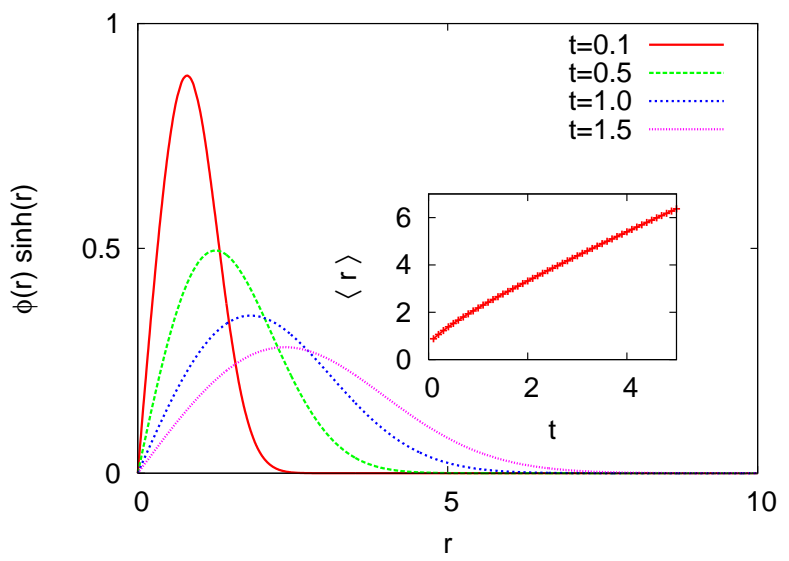

FIG. 3: (Color online) The solution $\phi(r, t)$ of the hyperbolic diffusion equation. Inset: Average distance from the origin $\langle r\rangle=\int r \phi(r) \sinh r d r$ increases linearly in time.

worthy and thus the decrease of $\left\langle d_{h}\right\rangle$ should not be taken as real.

For the random walk on a $2 \mathrm{D}$ flat surface, the walker has zero probability of escape by Pólya's theorem which states that the walk becomes transient for dimensions larger than two [1]. If we denote the probability density of finding a particle at a position $\mathbf{r}$ after time $t$ as $\phi(\mathbf{r}, t)$, the diffusion process is described by $\partial \phi / \partial t=\nabla^{2} \phi$. Solving this equation by Fourier transformation, one can see that the expected displacement from the origin is proportional to the square root of time in the $2 \mathrm{D}$ flat surface. The Laplace operator $\nabla^{2}$ is changed to the Laplace-Beltrami operator for the hyperbolic metric [15],

$$
\triangle=\frac{1}{\sinh r} \frac{\partial}{\partial r}\left(\sinh r \frac{\partial}{\partial r}\right)+\frac{1}{\sinh ^{2} r} \frac{\partial^{2}}{\partial \theta^{2}}
$$

and we get the solution of the hyperbolic diffusion equation, $\partial \phi / \partial t=\triangle \phi[11,16]$ :

$$
\phi(r, \theta ; t) \propto \frac{e^{-t / 4}}{t^{3 / 2}} \iint d r^{\prime} d \theta^{\prime} \phi\left(r^{\prime}, \theta^{\prime} ; 0\right) I(t, \rho) \sinh r^{\prime}
$$

with the distance $\rho$ between two positions $(r, \theta)$ and $\left(r^{\prime}, \theta^{\prime}\right)$, and $I(t, \rho) \equiv \int_{\rho}^{\infty}\left(s e^{-s^{2} / 4 t} / \sqrt{\cosh s-\cosh \rho}\right) d s$. The numerical integration is then performed to get the result presented in Fig. 3, which again shows the linear diffusion $\langle r\rangle \propto t$. We thus conclude that our different approaches, i.e., discrete random walks by triangle reflections on the Poincaré disk and the solution of the hyperbolic diffusion equation, unanimously confirm that the distance from the origin increases linearly in time. In comparison to $\langle d\rangle \sim \sqrt{t}$ for the $2 \mathrm{D}$ flat surface, the diffusion occurs much faster in the negatively curved surface.

We next study the diffusion of a tight-binding quantum particle in the heptagonal lattice. The quantum diffusion phenomena, and the existence and nature of the localization transition have been studied on regular lattice structures for a long time. Motivated by the intensive research

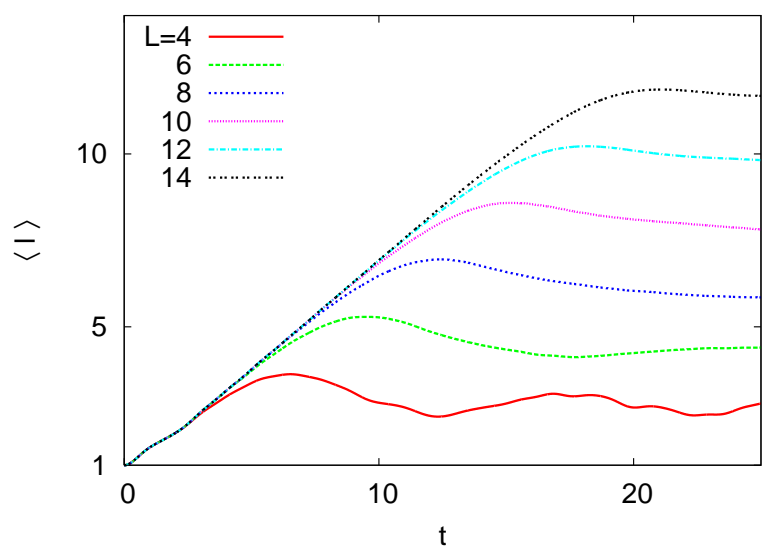

FIG. 4: (Color online) Diffusion of the tight-binding quantum particle on the heptagonal lattice. As the time $t$ evolves, the quantum particle diffuses toward the upper layers. Note that the linear regime extends as the size becomes larger.

interest of complex networks, recent years have observed the beginning of research on quantum mechanical systems put on the structure of complex networks [2, [3, 17]. We assume that the wave function is localized on the first layer containing seven points [see Fig. 1(a)]. The time evolution of the quantum particle is governed by the Schrödinger equation $i(\partial / \partial t)|\psi\rangle=\mathcal{H}|\psi\rangle(\hbar \equiv 1)$. By using the perfectly localized states as basis kets, we apply the tight-binding approximation that $\mathcal{H}_{i j}=1(0)$ if two points $i$ and $j$ are connected (not connected). The Schrödinger equation is then numerically integrated by the fourth order Runge-Kutta method. The spreading of the wave packet is measured by the average layer, $\langle l\rangle$, depicted in Fig. 4. Again, one can see clearly that the particle diffuses linearly in time as in the classical diffusion. The saturation comes from the finiteness of our lattice. It is to be noted that in the negatively curved heptagonal lattice, both the quantum and the classical diffusion exhibit the linear diffusion.

We then take an alternative approach to investigate the quantum diffusion problem, through the use of the mapping of the above tight-binding Hamiltonian to the one for a free particle. We note that the shift of energy by a constant amount does not change any measurable quantity and makes the transformation $\mathcal{H} \rightarrow \mathcal{H}-k I$ with a degree $k$ (the number of neighbors, e.g., $k=3$ for the heptagonal lattice) and the identity operator $I$. This simple transformation makes the Hamiltonian proportional to the lattice Laplacian, except for the outermost points where $k \neq 3$. In short, the tight-binding Hamiltonian can be phenomenologically treated as that of the free particle simulated on the discrete lattice, described by $i \partial \Psi / \partial t=\triangle \Psi$ with the wave function $\Psi=\langle\mathbf{r} \mid \psi\rangle$. Accordingly, if we substitute the time $t$ in the Schrödinger equation by the imaginary time $i t$, it takes exactly the same form as the diffusion equation. It is then straightforward to apply the previous result on classical diffusion 


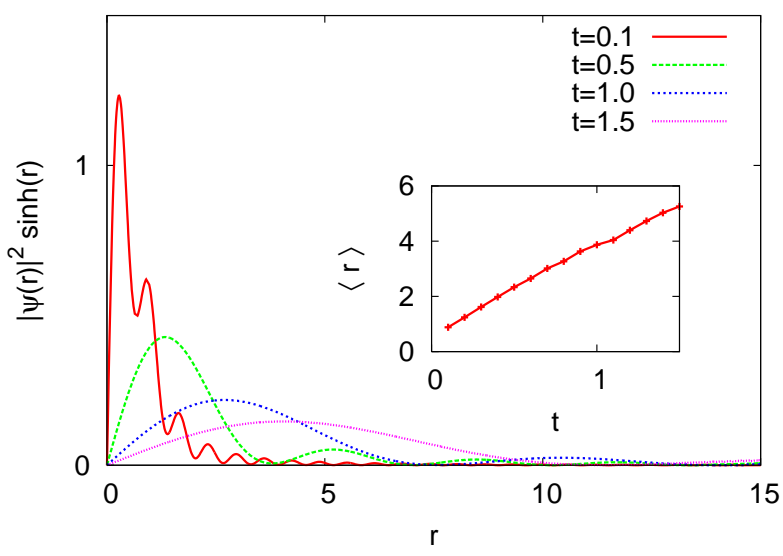

FIG. 5: (Color online) Solution of the quantum free particle Schrödinger equation in the negatively curved surface. Inset: Average distance from the origin $\langle r\rangle=\int r|\Psi(r)|^{2} \sinh r d r$ is shown to increase linearly in time.

in hyperbolic lattice (see Fig. 31) to obtain the propagating solution of the quantum particle on the hyperbolic plane [16] (see Fig. [5). It is revealed that the average distance again exhibits the linear diffusion property as shown in the inset of Fig. 5 in accordance with Fig. 4.

In the Euclidean or disordered structures, the quantum diffusion has been known to be faster than the classical one [2, 3]. In contrast, the classical motion now becomes comparable to the quantum one in speed on the negatively curved surface, with the aid of the geometrical drift. Some differences, however, seem to remain: The geometrical drift pushes the particle only in the outward direction from the starting point. Based on the report for tree structures, a quantum particle is believed to readily propagate back to the origin [18]. In fact, a classically diffusing particle acts somewhat like a flow, even if the initial direction may be chosen randomly, in the sense that it appears to have some nonzero velocity. If one introduces a real flow here, however, the flux would soon become negligible because of the exponentially increasing boundary. Our observation is qualitatively similar to the report for the Bethe lattice [11], since the angular movement is effectively suppressed in the long run. Yet it is still notable that the heptagonal lattice provides a better representation for the negatively curved surface, and that the numerical simulation could be taken with more ease by the hyperbolic tessellation technique explained above. One may expect that the transport can be enhanced by introducing a negative Gaussian curvature in biological or engineering applications.

In summary, we have investigated the classical and quantum diffusions in the heptagonal lattice, which is a discrete representation of the surface with a constant negative curvature. Even a classical particle has been shown to diffuse so fast that the average displacement is linearly proportional to time. The quantum diffusion has also been shown to exhibit the same linear diffusion behavior. Those results on discrete lattices were also confirmed in a continuum by solving a hyperbolic diffusion equation in real and imaginary times for classical and quantum diffusion, respectively.

\section{Acknowledgments}

This work was supported by the Korea Research Foundation Grant funded by the Korean Government (MOEHRD) with the Grant No. KRF-2005-005-J11903 (S.K.B.) and KRF-2006-312-C00548 (B.J.K.).
[1] J. Rudnick and G. Gaspari, Elements of the Random Walk: An Introduction for Advanced Students and Researchers (Cambridge University Press, New York, 2004).

[2] B. J. Kim, H. Hong, and M. Y. Choi, Phys. Rev. B 68, 014304 (2003).

[3] O. Mülken and A. Blumen, Phys. Rev. E 73, 066117 (2006).

[4] J. D. Noh and H. Rieger, Phys. Rev. Lett. 92, 118701 (2004); J. D. Noh and S.-W. Kim, J. Korean Phys. Soc. 48, S202 (2006).

[5] H. Shima and Y. Sakaniwa, J. Stat. Mech. (2006) P08017; J. Phys. A: Math. Theor. 39, 4921 (2006); L. R. A. Belo, N. M. Oliveira-Neto, W. A. Moura-Melo, A. R. Pereira, and E. Ercolessi, Phys. Lett. A 365, 463 (2007); F. Sausset and G. Tarjus, J. Phys. A: Math. Theor. 40, 12873 (2007).

[6] S. K. Baek and B. J. Kim, Europhys. Lett. 79, 26002 (2007).

[7] W. P. Thurston and J. R. Weeks, Sci. Am. 251, 108-120 (1984).
[8] J. W. Anderson, Hyperbolic Geometry (Springer-Verlag, London, 1999).

[9] D. Vanderbilt and J. Tersoff, Phys. Rev. Lett. 68, 511 (1992); N. Park, M. Yoon, S. Berber, J. Ihm, E. Osawa, and D. Tománek, ibid. 91, 237204 (2003).

[10] W. S. Kendall, Sém. Probab. (Strasbourg) 18, 70 (1984).

[11] C. Monthus and C. Texier, J. Phys. A: Math. Theor. 29, 2399 (1996).

[12] A. Karlsson, in Proceedings of a Workshop at the Schrödinger Institute, Vienna, 2001 (de Gruyter, Berlin, 2004), p. 459.

[13] H. S. M. Coxeter, Bull. Can. Math. 40, 158 (1997).

[14] M. J. Greenberg, Euclidean and Non-Euclidean Geometries: Development and History (W. H. Freeman, New York, 1993), 3rd ed.

[15] E. C. Young, Vector and Tensor Analysis, 2nd ed. (Marcel Dekker, New York, 1993).

[16] V. Banica, Commun. Partial Differ. Equ. 32, 1643 (2007).

[17] C.-P. Zhu and S.-J. Xiong, Phys. Rev. B 62, 14780 
(2000); O. Giraud, B. Georgeot, and D. L. Shepelyansky, Phys. Rev. E 72, 036203 (2005).

[18] A. M. Childs, E. Farhi, and S. Gutmann, Quantum Inf.
Process. 1, 35 (2002). 